\title{
Slowly progressive aphasia: three cases with language, memory, CT and PET data
}

\author{
D Kempler, E J Metter, W H Riege, C A Jackson, D F Benson, W R Hanson
}

\begin{abstract}
Three cases of slowly progressive speech and language disturbance were studied at various points post onset (three, five and 15 years respectively). Language, neuropsychological and brain imaging (computer tomography and positron emission tomography) evaluations were completed on all three patients. The data suggest that the syndrome of "progressive aphasia": 1) does not involve a uniform symptom complex; 2) does not necessarily develop into a full blown dementia syndrome; 3 ) varies greatly in rate of progression from case to case; 4) is associated with normal brain structure (on computer tomography); and 5) is associated with abnormal left temporal lobe metabolism as measured by fluorodeoxyglucose (FDG) positron emission tomography (PET). One patient had histological findings consistent with Alzheimer's disease at necropsy.
\end{abstract}

Speech Pathology, School of Medicine and School of Gerontology, University of Southern California, Los Angeles, California D Kempler

Gerontology Research Center, Baltimore, Maryland

E J Metter

VA Medical Center, Sepulveda, California W H Riege

VA Medical Center, Sepulveda and UCLA, California

C A Jackson

Reed Neurological

Institute, UCLA

California

D F Benson

VA Medical Center,

Sepulveda, California,

United States

W R Hanson

Correspondence to:

Dr Kempler, Speech

Pathology, OPD 2P52, Los

Angeles County/USC

Angeles County/USC

Medical Center, 1175

Aummings Street, Los

Angeles,

Received 20 November 1989 and in revised form

25 February 1989.

Accepted 14 March 1990
Approximately 29 cases of slowly progressive aphasia without dementia have been reported in the literature, ${ }^{12}$ but there remain unanswered questions about this syndrome. It remains to be seen whether this syndrome develops into a generalised dementia, ${ }^{1-7}$ and we have yet to determine its underlying neuropathology..$^{8-11}$ Additionally, little is known about its development (rate of progression) or the epidemiology.

The purpose of this report is to furnish more information about such patients. We have identified three individuals with progressive speech and language deficits of unknown aetiology. Our goals were: 1) to document via serial testing and through medical records the presence of a progressive language deficit, 2) to compare language problems with other areas of mental status, and 3) to investigate through neuroimaging the structural and metabolic bases for such disorders. A recent neuropathological necropsy report is included for one case. The three patients are described below.

\section{Method}

Language and Memory

The patients were tested with the Western Aphasia Battery (WAB), ${ }^{12}$ which includes measures of spontaneous speech (fluency and information content), auditory comprehension (yes/no questions, sequential commands), repetition, naming, reading and writing. Measures of praxis, drawing and calculation are also collected as part of the WAB. Scores are converted into a 10 point scale (10 = normal; $0=$ grossly deficient).

To evaluate other aspects of mental functioning, the patients were administered a neuropsychological battery which included standard administration of published tests, ${ }^{13-16}$ and an additional set of verbal and non-verbal memory tests designed to discriminate leftfrom right-hemisphere functions. ${ }^{1718}$ The verbal tests included:

1 Story recall. The subject listens to a short story (an Aesop fable of 141 words) and is asked to retell it immediately.

2 Sentence memory. The subject is asked to repeat well formed, semantically anomalous and grammatically anomalous sentences.

3 Picture recall. The subject is presented with nine line drawings on a single board and asked to describe each one. After 20 minutes, he is asked to describe the pictures from memory.

4 Immediate and delayed memory of visually presented (printed) words. The patient is presented with a series of 20 words on a tachistoscope and then asked to recognise the original words within a set of 40 items immediately after presentation and again twenty minutes (delayed) after presentation. Only a simple verbal or gestural indication of "yes" or "no" is required. Non-verbal tasks included both traditional tests of attention and reasoning ( $5,6,8$ below) as well as an array of nonverbal memory tasks developed by one of the authors (WHR). These were:

5 Block design. The subject is shown red and white blocks and asked to reconstruct seven printed designs. ${ }^{14} 15$

6 Ravens Coloured Progressive Matrices. The subject is shown a visual pattern with one part removed and asked to point to one of six pictured inserts which contain the correct pattern. ${ }^{13}$

7 Face Matching. The patient is presented with pictures of 20 famous people from each of three decades (1950s, 1960s, 1970s), and asked to point to 10 of them when named by the examiner.

8 Rhythm discrimination. The subject is asked to discriminate between like and unlike pairs of musical beats. ${ }^{16}$

9 Design recognition. The subject is shown a set of 20 slides of abstract paintings (by Vasarely) and asked to recognize the original set when shown a larger set of 40 paintings.

10 Auditory non-verbal recognition. The 
subject listens to a set of eight distinct bird calls through head phones and is asked to recognise the original set (by responding "yes" or "no") when listening to a larger set of bird calls. Recognition is tested immediately after presentation and then again 20 minutes later (delayed condition).

11 Pattern reconstruction. The patient is shown a checkerboard pattern card containing a design of red dots for five seconds, asked to wait five seconds, and then asked to reconstruct the red-dot pattern on a blank checkerboard card.

Performance on these tests are compared with the performance of 200 normal controls, and converted into $\mathrm{Z}$-scores. For our purposes, $z$-scores between -1 and -2 indicate a mild impairment, between -2 and -3 indicate a moderate impairment, and greater than -3 a severe impairment.

\section{Neuroimaging}

To discover neurofunctional information, patients were studied with (F-18) fluorodeoxyglucose (FDG) positron emission tomography $\left(\right.$ PET) on a Neuro-ECAT ${ }^{1920}$ in a resting state with eyes and ears unoccluded. Patients lay on the scanner bed in a darkened room, listening to ambient room noise and were injected intravenously with $18.5 \times 10^{10}$ to $37 \times$ $10^{10}$ becquerels ( 5 to $10 \mathrm{mCi}$ ) of $18-\mathrm{F}$ fluorodeoxyglucose, followed by the drawing of arterialised venous blood samples. After 40

\section{Figure 1 Diagram of} regions analysed for structural damage and glucose metabolic rates. Regions 1 and 2 are high frontal; 3 and 4, low frontal; 5 and 6, Broca's; 7 and 8, parietal; 9 and 10, Wernicke's; 11 and 12, temporal; 13 , occipital; 14 caudate; and 15, thalamus. (Reprinted from Kempler et al, Disconnection and Cerebral Metabolism, Archives of Neurology 1988;45:275-9.

Copyright 1988, American Medical Association.)

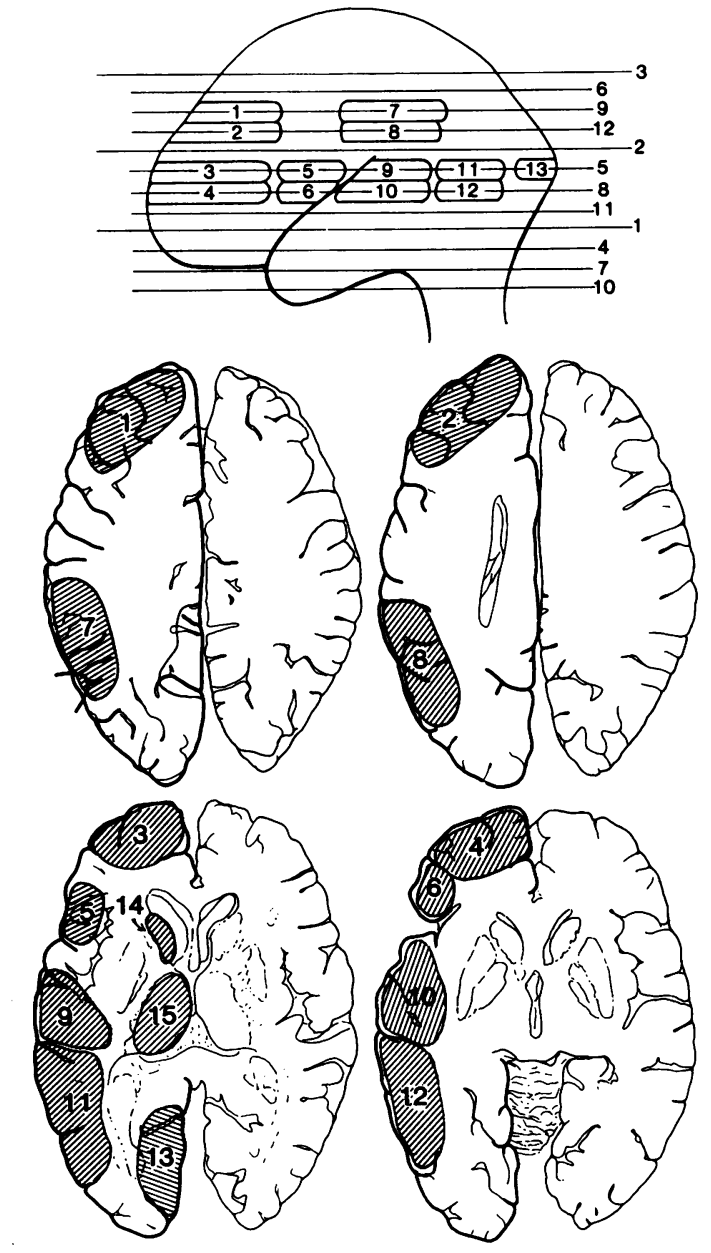

minutes, scanning was initiated. Values of local cerebral metabolic rate for glucose (LCMRGlc) were calculated as previously described. ${ }^{1920}$ Fifteen cerebral regions from each hemisphere (fig 1) were outlined on a video monitor using an interactive program, and regional LCMRGlc values were derived in milligrams of glucose per $100 \mathrm{~g}$ of tissue per minute. Region of localisation was based on the atlas of Matsui and Hirano. ${ }^{21}$ To reduce the number of comparisons, cortical regions from adjacent tomographic levels were averaged to give nine measures per hemisphere (for example, regions 1 and 2 combined to form a "high frontal" region, 3 and 4 to form a "low frontal" region, and 5 and 6 to form Broca's area). The LCMRGlc values of aphasic patients' right hemispheres differ little from those of healthy controls but may show interindividual variation; therefore, left-right ratios for homologous regions were calculated and used in analyses. Ratios of less than 0.90 were considered abnormal for each regional ratio when compared with 22 normal controls.

Each subject also had non-contrast CT scan (on either a Picker 1200 SX or GE 8800) with scanning in the same plane as PET. The same regions as measured for glucose metabolism were rated. In addition, the anterior internal capsule, posterior internal capsule, insula, and lenticular nuclei were rated. The regions of interest were rated using a five-point scale ( 0 , normal; 1 , atrophy; 2 , structural damage with no tissue loss; 3 , structural damage with partial tissue loss; and 4, structural damage with complete tissue loss) by a neuroradiologist who was blind to the project and by one of the authors (EJM). Regional scores showed a 90\% agreement between raters, and the two ratings were averaged to obtain an estimate of the degree of structural damage. The three cases are presented individually below.

\section{Case 1}

This is a left-handed male with a history of difficulty in remembering words, names and faces that has progressed very slowly for approximately 15 years before our first evaluation. At the most recent testing he was 59 years old. He has been a puzzle to his physicians, and has accumulated an impressive list of diagnoses, including chronic endogenous or possibly psychotic depression, sarcoid brain lesion, slow growing glioma, toxin exposure, Alzheimer's Disease, hippocampal damage, and Pick's disease. Adequate medical records have been obtained over an 18 year period to document the progressive decline of function.

His early educational and occupational functioning were good. He holds several degrees and enjoyed a successful sales career. A diagnosis of sarcoid node of the shoulder was confirmed by cervical biopsy. Word-finding problems were first noted in the early 1970 s and progressed until they interfered with his ability to work in 1977-78, at which point he was laid off. Since then his ability to find words, to understand spoken language, and to recognise familiar faces has deteriorated. He now participates in the compensated work therapy 
Figure 2 Performance on the Western Aphasia Battery subtests for the three patients.

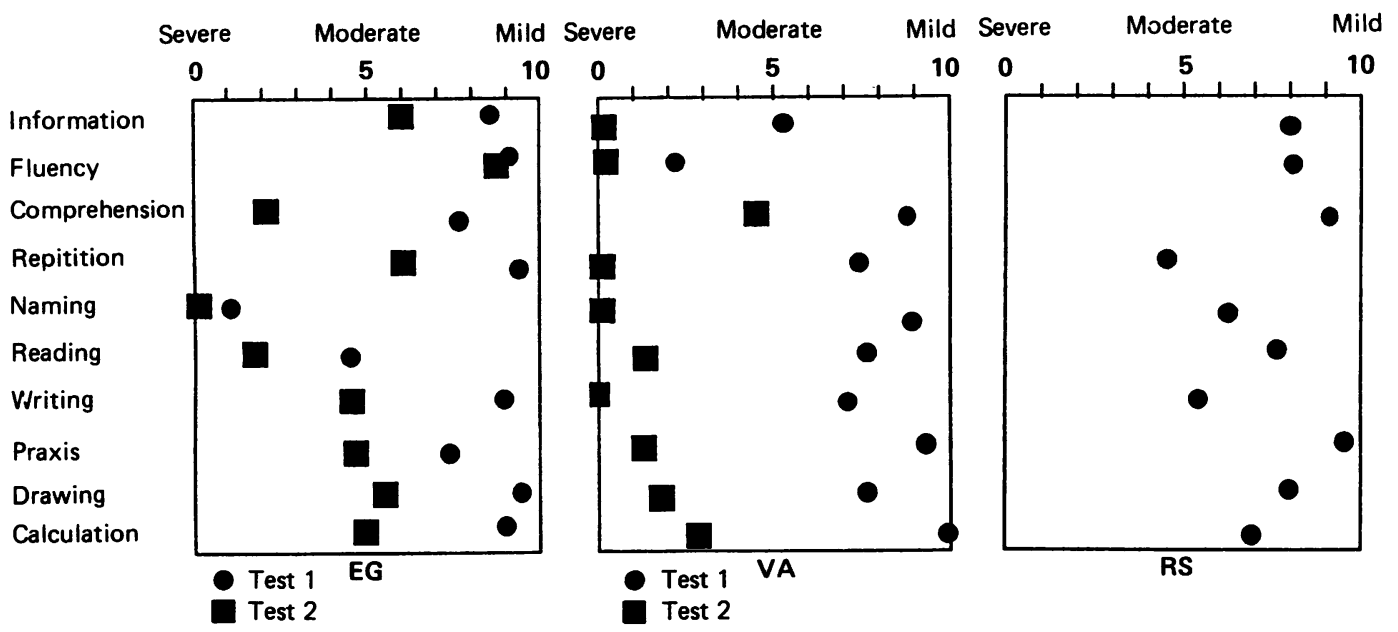

programme at a VA Medical Center, where he performs unskilled labour. Through this entire period, neurological examinations have remained normal. He continues to drive in the Los Angeles area, keeps appointments and appears to have insight into his current problems.

\section{Test data}

Language test data are available from 1984 and 1986 (fig 2). Naming was his most pronounced language deficit; he was unable to name any of 20 objects presented on the WAB. Spontaneous speech was moderately fluent and informative, but marked by many episodes of anomia at both testing sessions. His description of the picture from the WAB (which shows a family picnic at a lakeside and includes a tree, house, car, dog, sailboat, a man and woman near the picnic basket, and several children playing near the lake) elicited "...there is an animal. I forget what you call that. Not an a pen or a pencil. I forget. Here's a car. There's a house. I forget what this is. I forget what that is.
I know what that is but I don't know the word. It's near a, not a movie, a water. It's near water." Comprehension was mildly impaired at the first session, and severely impaired two years later. Repetition appeared relatively preserved, but also showed some decline over time. Reading for comprehension was moderate-severely impaired at both sessions but writing deteriorated from appropriate and legible to moderately impaired between the two test dates. All language measures except "fluency" showed decline over the two year period.

Neuropsychological data are available from three sessions over a five year period, beginning three years before the first language test data (fig 3). He performed within the normal range on nonverbal tests of problem solving (Ravens Progressive Matrices, WAIS block design), at all three sessions although some decline in function can be observed between initial and third sessions. He showed mildly impaired performance on recognition of famous faces. Interestingly, prosopagnosia was one of his

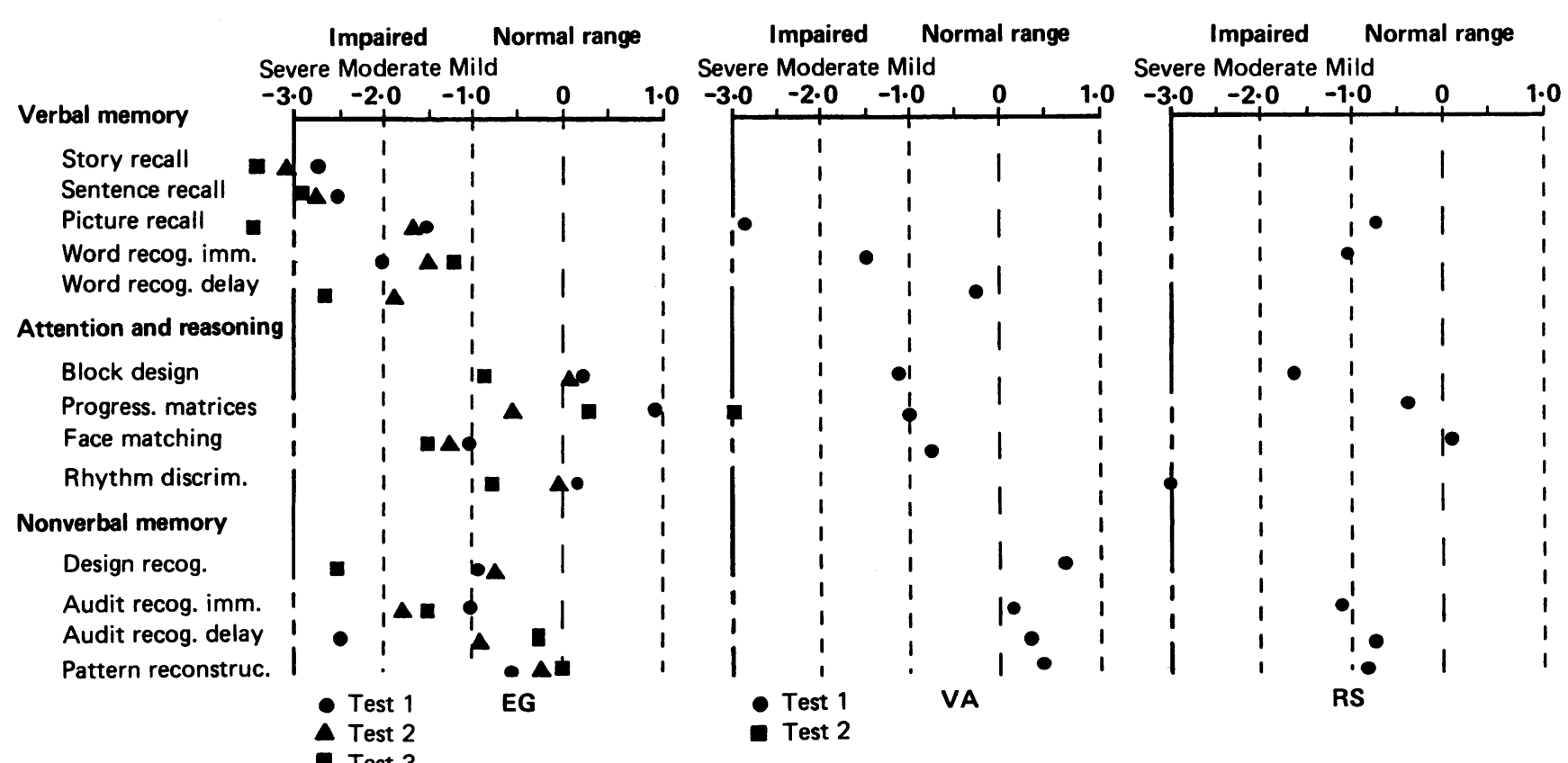

Figure 3 Performance on the Neuropsychological Test Battery for the three patients. 
Table 1 Left-right metabolic ratios for nine cerebral regions

\begin{tabular}{llllllllll}
\hline Subject & HiF & LoF & Broca & Prtl & Wern & Temp & Caud & Thal & Occ \\
\hline 1 & 0.92 & 0.90 & $0.85^{\star}$ & $0.81^{\star}$ & $0.80^{\star}$ & $0.77^{\star}$ & 1.01 & 1.00 & 0.92 \\
2 & $0.75^{\star}$ & $0.80^{\star}$ & $0.94^{\star}$ & $0.73^{\star}$ & $0.83^{\star}$ & $0.77^{\star}$ & $0.70^{\star}$ & $0.80^{\star}$ & 0.96 \\
3 & $0.85^{\star}$ & $0.87^{\star}$ & $0.83^{\star}$ & $0.77^{\star}$ & $0.74^{\star}$ & $0.68^{\star}$ & 0.91 & $0.86^{\star}$ & 0.96 \\
\hline
\end{tabular}

^Significant hypometabolism (greater than two standard deviations below the normal mean).

major complaints and was consistently demonstrated in daily interactions, where the patient was unable to recognise the examiner, except when he was seen in the familiar context of the testing office. Non-verbal memory ranged from the low normal to impaired range but showed no particular pattern of decline. For example, on two measures he improved over the five year period, while he showed marked decline on only one measure (design recognition). The consistent and severe impairments were observed in tests of verbal memory, where he scored in the impaired range and demonstrated a general decline in three separate tests over the five year period. The overall neuropsychological picture is not one of continuous cognitive decline; less than half of the neuropsychological measures showed decreased performance over a five year period. This contrasts with language measures which showed marked decline in all measures except fluency.

\section{Brain structure and function}

Comparable slices of a non-contrast CT and PET scans are presented in fig 4. A CT scan documented slight generalised atrophy, with slightly enlarged ventricles, and sulci, particularly in the left perisylvian area. A PET scan documented significant hypometabolism in the left posterior temporal and parietal areas, as well as inferior frontal (Broca's) areas. Quantitative analysis of metabolic data confirm four perisylvian regions of significant hypometabolism: Broca's, Parietal, Wernicke's and Temporal (table 1).

Case 2

This is a 71 year old, right handed male with a three year history of a decline in word-finding and other speech production abilities. He had been employed as a farmer, a dry food packer and in real estate. He failed to pass a test for a real estate broker's license in early 1984, which prompted the initial concern, and soon after was forced to retire due to his deteriorating

Figure $4 C T$ and PET scans of patient 1.CT scan showed slight, generalised, bilateral atrophy with slightly enlarged ventricles and sulci. PET scan demonstrated significant metabolic asymmetry, particularly pronounced in the area of the left temporal lobe. Thalami and caudate appear symmetrical. condition. His major complaints centred around communication: his voice was weak, he had difficulty speaking quickly, and he often had trouble getting words out. He also complained of stuttering, memory impairments, and trouble with handwriting.

Initially, his speech was described as "slow dsyprosodic hypophonic verbal output with stutter." The presence of a somewhat shuffling gait, mild rigidity of the extremities and a general slowness were consistent with a diagnosis of Parkinson's disease. However, the stuttering behaviour and severe anomia were felt to be atypical of Parkinson's disease (but see reference ${ }^{22}$ ). Subsequent evaluations by two neurologists and a neurolaryngologist revealed similar findings, all mentioning the diagnosis of Parkinson's disease, and simultaneously noting the atypical presentation. Treatment with dopaminergic drugs had no notable effect on the symptoms and neither a resting tremor nor festination were observed. Tongue movement was relatively rapid compared to other patients with basal ganglia disease. Although speech production (low volume, harsh breathy voice, monotone) was consistent with extrapyramidal disease, and typically Parkinsonian, the presentation of severe speech disturbance, with only mild (or inconsistent) other Parkinsonian symptoms led to some questioning of the diagnosis. Over the three years that he was followed, his speech continued to deteriorate so that it became a whisper of single words and then undifferentiated phonation. At this point he was still able to supplement his speech with writing. He remained ambulatory with otherwise only mild Parkinsonian features.

\section{Test data}

Initial language and memory evaluation were completed approximately three years after speech and language symptoms were first noticed. His language symptoms were mild-tomoderate, with particular deficits in repetition, and difficulty understanding sentences. Confrontation naming ( $95 \%$ correct) gives a possibly false impression of preserved word finding and fluency. In conversation he was often unable to generate sentences at all, and demonstrated many apparent word finding difficulties. For example, the Cookie Theft picture from the Boston Diagnostic Aphasia Examination elicited this narrative: "The mother is....dishen, and.....the girl, the boy is getting cookie, and uh, .....the girl is uh, so- sosodgering, and oneladdeh- oneladdeh- oneladdeh-, and......boy's, boy's on the stool, and uh, I don't know. It's a kitchen, and drapes, and window, and out in yard, cupboards."

Retesting two years later revealed significant declines in all areas of language: verbal output 
was restricted to periodic undifferentiated phonation in response to questions, making spontaneous speech, naming, and repetition impossible to evaluate; comprehension for simple material remained good, but comprehension of complex material (for example, sequential commands) was severely impaired; reading showed continued dissolution and writing was impossible (fig 2).

Neuropsychological testing was only completed once since he was unable to come to the clinic at the two year follow up point (fig 3). Tests which required extensive verbal production (sentence and story recall) were not completed and the other tests of verbal functions showed variable performance. Tests of nonverbal problem solving were near normal/borderline and non-verbal memory was superior. Only the Ravens Progressive Matrices was repeated at the second interview, and revealed impaired non-verbal reasoning (13 of 36 correct; approximately tenth percentile for age from published norms).

\section{Brain structure and function}

Figure 5 shows CT scan showing mild generalised atrophy, and a PET scan exhibiting marked focal hypometabolism in the left frontal, superior temporal, inferior parietal, and thalamic regions, as well as the insula and head of caudate. Relatively spared regions included inferior frontal and inferior and middle temporal areas.

\section{Case 3}

This is a 63 year old right handed male with a history of word-finding difficulties which developed over a period of five years. Before this, the patient was a successful engineer. Initial differential diagnosis included investigation for brain tumour, but all confirming tests were negative. The patient's complaints centred around anomia, or, in his words, "the dropping of the words. It is losing it, and uh, I like great, great, and so forth."
Test data

Language and memory testing took place approximately five years after anomia was first noticed. Verbal output was fluent, but paraphasic in all tasks, including repetition and naming. The Cookie Theft picture elicited the following utterances: "I see the ski-, the uh, the bis, the water is dran, lon, don, lay-, and flow-is flowing in the wall, identify. Kids are- cookie, jar, sneaking 'em I guess, and that- that's..." Comprehension was relatively preserved, with some deficit noted in understanding sequential commands. Writing contained paraphasic errors, similar to those noted in speech production. Nonverbal intelligence (Ravens Matrices) appeared good, although a mild impairment was observed in block design and a more severe impairment in rhythm discrimination. Memory for both auditory and visual information was in the normal range. Retesting was not accomplished before the subject's death. The relatively better performance on the Ravens Matrices and nonverbal memory when compared with block design and rhythm discrimination suggests that the nonverbal deficit may involve construction and sequencing more than perception or general problem solving ability. Language and Neuropsychological test data are presented in figs 2 and 3.

\section{Brain structure and function}

CT scans were uniformly negative but the last CT scan did reveal mild-moderate generalised atrophy, greatest in the left perisylvian area. The PET scan demonstrated left temporal hypofunction, which appeared to extend to the left frontal areas and thalamus (fig 6).

The patient died approximately two years after this testing (seven years after symptoms first appeared). At necropsy examination* the brain weighed 1300 grams, the hemispheres appeared symmetrical without evidence of

«The authors gratefully acknowledge the contribution of Barbara Zaias for providing the neuropathological evaluation.
Figure 5 CT and PET scans of patient 2. CT scan demonstrated slight generalised atrophy. PET scan revealed asymmetry with significant hypometabolism throughout much of the left hemisphere, particularly marked in frontal and temporal areas.

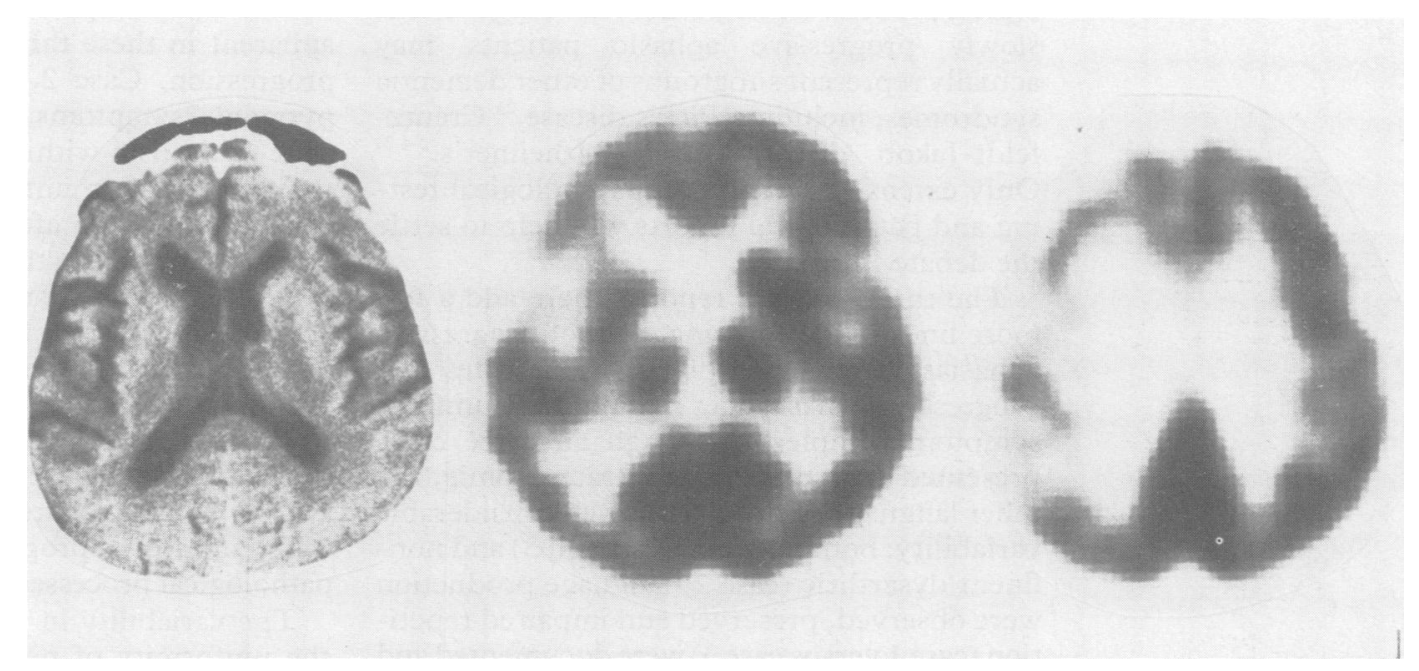


Figure $6 \quad C T$ and PET scans of patient 3. CT scan showed bilateral atrophy in the perislyvian area (left greater than right) and mild-moderate generalised atrophy. PET scan demonstrated prominent metabolic depression in the left temporal area, as well as left frontal region and thalamus.

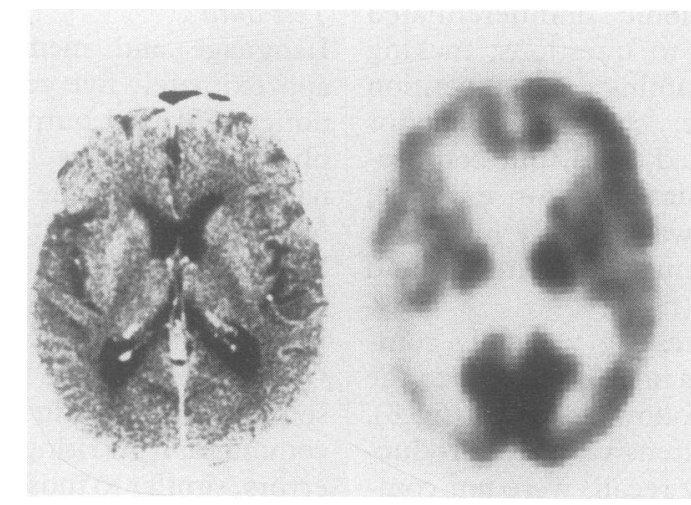

gross abnormality or significant atrophy. Microscopic examination, however, revealed abundance of neuritic plaques and neurofibrillary tangles spread diffusely throughout the cerebral cortex. This ranged from 15-35 plaques per $10 \times$ field in the frontal cortices. Neurofibrillary tangles were present in most cortical areas but were less numerous. Granulovacuolar degeneration was present in the hippocampal cells. The neuropathological findings were consistent with a diagnosis of Alzheimer's disease.

\section{Discussion}

These three cases differ from one another clinically. One patient presented with a slowly (15 year) progressing language and verbal memory deficit which also progressed to involve visual memory, leaving non-verbal problem solving unaffected; the second patient presented with a three year progression which included anomia and hypokinetic dysarthria, with eventual involvement of non-verbal problem solving; and the third case presented with a five year course of progressive anomia, with some other symptoms of left-hemisphere dysfunction, including a calculation deficit, and poor perception of rhythm, leaving most other functions intact.

Mesulam"15 has argued that "progressive aphasia" be reserved for those patients who do not eventually develop other cognitive deficits. Further, he predicts that these patients may prove to be suffering from a unique pathological process. Other authors have argued that slowly progressive aphasic patients may actually represent subgroups of other dementia syndromes, including Pick's disease, ${ }^{23}$ Creutzfeldt-Jakob disease ${ }^{10}$ and Alzheimer's. ${ }^{2-4} 24$ Only extensive serial neuropsychological testing and pathological reports will help to settle the debate.

The three patients reported here add a few more lines to the evolving story of progressive aphasias. A review of our cases indicates that progressive aphasia does not involve a uniform symptom complex. Although all three cases presented with at least moderate anomia, the other language symptoms showed considerable variability: both fluent (cases 1 and 3) and nonfluent/dysarthric (case 2) language production were observed, preserved and impaired repetition (case 1 versus case 3 ) were documented and auditory comprehension varied greatly between patients and over time. The aphasia categories diagnosed (by WAB) in these cases also demonstrates the variability: case $1=$ Anomic aphasia; case $2=$ Broca's aphasia; case 3 = Conduction aphasia.

Although all three patients presented initially with aphasic symptoms, and aphasia continued to be their primary complaint throughout the course of study, other neuropsychological deficits were observed. Several areas of cognition are typically impaired in aphasia, including calculation and praxis. ${ }^{25}$ The range and severity of these non-language cognitive impairments also varied in our three patients. Verbal memory (to the extent that it was testable) was impaired in two patients. Note that following the instructions and responding appropriately to these tests requires relatively intact language comprehension and production skills, and poor performance here might only reflect the aphasia, not an independent memory deficit. Calculation and praxis were measured via the WAB and demonstrated a range of performance: praxis ranged from $45 \%-95 \%$ correct; calculation ranged from $66 \%-100 \%$; and construction (measured by drawing) ranged from $73 \%-97 \%$ correct. Nonverbal measures of memory and problem solving also revealed variable performance, ranging generally from mild impairment to superior performance.

As we move further from language tests, and those which require language output, performance for all three subjects is in the normal or near-normal range. As we include tests which rely on similar cognitive or (left hemisphere) neural substrates, such as praxis, rhythm discrimination and calculation) we begin to see variable performance decrements. As we then progress to language, we see impairment in all three patients. In sum, the range of nonlinguistic neuropsychological deficits is limited and less severe than the language disturbance, but impairments can be documented nonetheless. This, as has been pointed out by others, ${ }^{2}$ is no different from what you find in traditional focal aphasic patients with left hemisphere infarcts who present with a range of verbal memory deficits, apraxias and acalculias, but retain basic nonverbal reasoning powers.

One other aspect of variability which is apparent in these three cases is in the rate of progression. Case 2, the patient with extrapyramidal symptoms, appeared to progress to a state of mutism within five years: he was seen three years after initial symptoms appeared, and within a year after that was too severely impaired to come to the hospital for testing and was found to be fully nonverbal two years later. In contrast, case 1 had a very slow course. Fifteen years after the initial symptoms, he was still physically strong, driving independently, performing volunteer work, and dropping by the author's office weekly to inquire (verbally) after a potential cure for his problems. The different rates of progression suggests separate pathological processes.

The variability in symptoms contrasts with the uniformity of neuroimaging information. 
The CT data presented here essentially agree with past reports ${ }^{1124}$ which have found normal or mild left-sided atrophy on CT without evidence of neoplasms, infarcts, etc. Brain function, as measured by PET, demonstrated similarities and differences across cases. The common feature to all three cases was left temporal hypometabolism, and normal right hemisphere glucose metabolism, again confirming past research with similar cases. ${ }^{2}$ However, our cases differed in the degree of hypometabolism in non-temporal areas of the left hemisphere. The case with the slowest progression of symptoms (case 1) showed the most circumscribed area of hypofunction, limited essentially to the left temporal lobe. The case with Parkinsonian features (case 2) showed more widespread left hemisphere hypometabolism, including temporal, parietal, insular, thalamic and caudate regions. $\dagger$ The final case (case 3) demonstrated depressed function in all areas of the left hemisphere except the occipital lobe and the caudate. It was this case who came to postmortem and had neuropathological findings consistent with Alzheimer's disease. The common finding of left temporal metabolic dysfunction in the absence of obvious structural damage demonstrates the general point that observable structural brain damage is not a prerequisite to persisting aphasic symptoms. It appears that glucose metabolic brain dysfunction in the left temporal lobe affects behaviour much like abnormalities of brain structure (for example, stroke) in the same regions.

The question of whether these cases represent a unique entity or are variants of other known diseases can be raised. These cases are not typical presentations of known diseases, nor do they share a uniform symptom complex sufficient to warrant proposing a separate syndrome for all three. Rather, they may be atypical variants of known disease processesvariants with a particularly aphasic presentation. Pick's disease is an unlikely diagnosis for any of our cases considering the absence of personality and behavioural disorders. Vascular aetiologies are also unlikely considering the negative CT findings and the lack of a stepwise progression.

Atypical Parkinson's disease is a possible diagnosis for case 2, although the aphasia and lack of several cardinal Parkinsonian features have made that diagnosis questionable. Alzheimer's disease has been the most common diagnosis for patients with progressive aphasia, and necopsy data from case 3 (consistent with a diagnosis of Alzheimer's disease), force us to consider a diagnosis of atypical Alzheimer's disease at least in this one instance, despite the lack of strong behavioural evidence of Alzheimer's dementia. None of these known disease processes appears to be useful in describing case 1 who presented with the longest

†The PET pattern typically associated with Parkinson's disease (PD) is that of uniformly lower than norma measurements across all regions. ${ }^{26}$ The pattern observed in case 2 shows more focal findings (left/right asymmetry) but a diagnosis of PD cannot be excluded on these findings alone. history, the most severe, specific aphasic symptoms, and relative preservation of most nonverbal functions after 15 years of symptom progression. Although this case might be an atypical presentation of Alzheimer's disease, the longstanding uneven neuropsychological profile and the particularly protracted course make $\mathrm{AD}$ an unsatisfying diagnosis. Perhaps in this case, we have documented a more unique form of progressive aphasia, a disorder which is behaviourally and neurologically unique from other dementias.

1 Mesulam M-M. Slowly progressive aphasia without generalized dementia. Ann Neurol 1982;11:592-8.

2 Poeck K, Luzzatti C. Slowly progressive aphasia in three patients: The problem of accompanying neuropsychological deficit. Brain 1988;111:151-68.

3 Foster NL, Chase TN. Diffuse involvement in progressive aphasia. Ann Neurol 1983;13:224-5.

4 Kirshner HS, Webb WG, Kelly MP, Wells CE. Language disturbance; An initial symptom of cortical degenerations disturbance; An initial symptom of cortical degeneration

5 Mesulam M-M. Primary progressive aphasia-differentiation from Alzheimer's Disease. Ann Neurol 1987;22 533-4.

6 Neils J, Barrett E. The evolution of slowly progressive aphasia. Paper presented at the annual meeting of the American Speech-Language-Hearing Association, New Orleans, November, 1987.

7 Pogacar S, Williams RS. Alzheimer's disease presenting as slowly progressive aphasia. Rhode Island Medical Journal 1984;67:181-5.

8 Chawluk JB, Mesulam M-M, Hurtig H, et al. Slowly progressive aphasia without generalized dementia: Studies with Positron Emission Tomography. Ann Neurol 1986;19:68-74.

9 Kirshner HS, Tanridag O, Thurman L, Whetsell WO. Progressive aphasia without dementia: Two cases with Progressive aphasia without dementia: Two cases with focal spongiform degeneration. Ann Neurol 1987;22: 527-32.

10 Mandell AM, Alexander MP, Carpenter S. CreutzfeldtJakob disease presenting as isolated aphasia. Neurology 1989;39:55-8.

11 Morris JC, Cole M, Banker BQ, Wright D. Hereditary dysphasic dementia and the Pick-Alzheimer spectrum. Ann Neurol 1984;16:455-66.

12 Kertesz A. The Western Aphasia Battery. London, Ontario University of Western Ontario, 1980.

13 Raven JC. Coloured Progressive Matrices. London: HK Lewis, 1956. New York: Psychological Corporation, reprinted, 1978 .

14 Wechsler D. Wechsler Adult Intelligence Scale. New York: Psychological Corporation, 1955

15 Wechsler D. WAIS-R manual. New York: Psychological Corporation, 1981.

16 Seashore CE, Lewis D, Saetveit DL. Seashore Measures of Musical Talents (Rev ed). New York: Psychological Corporation, 1960.

17 Riege WH, Harker J, Metter EJ. Clinical validation: Brain lesions and brain imaging. In: Poon LW, ed. Handbook of clinical memory assessment in older adults. Washington, DC: American Psychological Association Press, 1986:314-36.

18 Riege WH, Metter EJ, Hanson W. Verbal and nonverbal recognition memory in aphasic and nonaphasic stroke patients. Brain and language 1980;10:60-70

19 Hoffman EJ, Phelps ME, Huang SC. Performance evaluation of a positron tomograph designed for brain imaging. $J$ tion of a positron tomograph

20 Phelps ME, Huang SC, Hoffman EJ, et al. Tomographic measurement of local cerebral metabolic rate in human with (F18) 2-fluoro-2-deoxyglucose: Validation of method. Ann Neurol 1979;6:371-88.

21 Matsui T, Hirano A. An atlas of the human brain for computerized tomography. Tokyo, Igaku-Shoin Ltd, 1978

22 Canter GJ. Observations on neurogenic stuttering: a contribution to differential diagnosis. Br J Dis Comm 1971 6:139-43.

23 Holland AL, McBurney DH, Moossy J, Reinmuth OM. The dissolution of language in Pick's disease with neurofibrillary tangles: A case study. Brain and Language 1985;24:36-58.

24 Gordon B, Selnes O. Progressive aphasia "without dementia": evidence of more widespread involvement. Presented at the 36th annual meeting of the American Academy of Neurology, Boston, Mass: April 8-14, 1984.

25 Kaplan E, Goodglass H. Aphasia-related disorders. In: Sarno MT, ed. Acquired Aphasia. New York: Academic Sarno MT, ed. Acgui

26 Metter EJ, Riege WH, Kameyama M, Kuhl DE, Phelps ME. Cerebral metabolic relationships for selected brain regions in Alzheimer's, Huntington's and Parkinson's Diseases. Journal of Cerebral Blood Flow and Metabolism 1984;4:500-506. 